\title{
Anesthesia Protocol Procedures for Hemodynamic Diagnostic and Interventional Cardiology
}

\author{
Leonardo Milella* \\ UOC Head of Anesthesia and Intensive Care TI-CCH Pediatric Hospital "John XXIII", Italy
}

Submission: August 28, 2018; Published: October 09, 2018

*Corresponding author: Leonardo Milella, General Neonatal and Pediatric Anesthesia and Intensive Care Pediatric Hospital "Giovanni XXIII"Viale Amendola 207, 70121-Bari, Italy, Tel: +39-080-559-6624; Fax: +39-080-559-6814, Email: dino.milella@virgilio.it

\begin{abstract}
The procedures of hemodynamic diagnostic and interventional cardiology require anesthesia care with general anesthesia in most cases with extubation and wake up of the patient at the end of the procedure This anesthetic protocol is applied to a patient population scheduled for routine procedures and / or urgency; anyway for particular cases and particular hemodynamic procedures dedicated anesthetic procedural thecniques are required.
\end{abstract}

Keywords: Pathosis; Trauma; Cortical bone; Deformities; Etiologic

\section{Introduction}

The use of sugammadex is routine as a drug reversal of the action of the muscle relaxant administered during anesthesia care it must be consulted in all those situations where a failed intubation or technical difficulties that delayed the same can lead to rapid deterioration of the state in cardiovascular patients as complexcases, premature, malformed babies and / or heart disease. In this sense, our experience leads us to consider sugammadex as a lifesaving medication and specific antidote [1].

\section{Special Cases}

Syndromic patients, malformed, famously presenting difficult intubation: Availability in the cath lab of fiberoptic bronchoscope and / or video laryngoscope; flexible measuring $2.8 \mathrm{~mm}, 3.5 \mathrm{~mm}, 5 \mathrm{~mm}$ with 3, 3.5 pipes,4, 5 pre-mounted on flexible of adequate gauge [2].

\section{Not Myoresolution}

Rapid induction with midazolam; propofol; TPS, sevoflurane, ketamine ie related and guided to age and weight. Laryngeal topical anesthesia with xylocaine $2 \mathrm{mg}$ / $\mathrm{kg}$ (optional) Intubation needs a sedated patient. Rocuronium from 0.9 to $1.2 \mathrm{mg} / \mathrm{kg}$ after certain intubation [3].

\section{Monitoring (similar for all procedures)}

Induction of anesthesia: Sp02 Heart Rate NIBP. After cannulation of the vessels: Right Atrial Pressure right ventricular pressure. Left Atrial Pressure (if required for he procedure) left ventricular pressure-invasive arterial blood pressure. EGA from arterial blood sample.

Patients Hyperflow from Left Right Shunt With or without Pulmonary Hypertension

\section{Patient weight $<5 \mathrm{~kg}$}

Premedication and research venous access if you do infuse suspend feeding three hours before the pro- tough you do not need premedication of the patient if present cannulation of the umbilical vein induce anesthesia from such access and continue anesthesia from such access, which will be removed at the end of procedure. If the peripheral venous access is difficult to obtain, induce anesthesia with sevoflurane $3.5-4 \%$ (titrate). Do not make more than two attempts to cannulate a peripheral vein or external giugular vein

then find a central venous access [4-7].

\section{Anesthesia}

a. Induction: Midazolam 1-2 $\mathrm{mg} / \mathrm{kg}$ in slow. Bolus Fentany $15 \mathrm{mcg} / \mathrm{kg}$. Rocuronium: $1.2 \mathrm{mg} / \mathrm{kg}$ for induction; It proceeds to intubation between 120 and180 seconds after the administration of the muscle relaxant. Rocuronium $1 \mathrm{mg}$ / kg before the puncture to cannulation of the vessels.

b. Retention: The maintenance should be started immediately after intubation TIVA: midazolam 2 to $8 \mathrm{mcg}$ / kg / min. Rocuronium $1 \mathrm{mg} / \mathrm{kg} / \mathrm{h}$ every 40 minutes. 
Sevoflurane $1-3 \%$. Suspension of anesthesia 10 minutes before the anticipated end of the procedure.

\section{Sugammadex}

The administration of sugammadex is provided in all cases in which, after 10 minutes from anesthesia suspension has not had an adequate restoration of spontaneous ventilation; in patients of this weight is not driven TOF because of reliability problems of reading in the premature infant or low weighting patients.

a. Failure Intubation: Sugammadex: $16 \mathrm{mg} / \mathrm{kg}$ in 30 seconds with restoration of spontaneous ventilation nea within 180 seconds. If not resume spontaneous breathing within 180 secondaryrepeating: Sugammadex: $6 \mathrm{mg} / \mathrm{kg}$ then: It repeats protocol as special cases.

b. Extubation Routine: Sugammadex: 2-4 mg / kg bolus in a minute with restoration of ventilation spontaneous within 180 seconds.

\section{Patient weight $<10 \mathrm{~kg}>5 \mathrm{~kg}$}

Premedication and research venous access, suspend eventual supply by 12 hours Suspend administration of water from 4 hours. Midazolam 0.09 to $0.1 \mathrm{mg} / \mathrm{kg}$ + droperidol 1.25 $\mathrm{mg}$ rectally $30 \mathrm{~min}$ - utes you before entering the cath lab. If the peripheral venous access is hard to come by, induce anesthesia with sevoflurane $3.5-4 \%$ (titrate). Do not make more than two attempts to cannulate a peripheral vein or external giugular vein then find a central venous access [8-11].

\section{Anesthesia}

a. Induction: Midazolam 1-2 mg / $\mathrm{kg}$ in slow bolus Rocuronium $1 \mathrm{mg} / \mathrm{kg}$ for induction. Rocuronium $0.3 \mathrm{mg}$ prior to cannulation of the vessels.

b. Retention: Except that in particular cases the maintenance procedures should be started immediately after the arterial cannulation, or immediately in hypertensive patients (eg., aortic coartation ) or in patients in whom we proceed to surgical cannulation of the vessels. TIVA: midazolam 2 to $10 \mathrm{mcg} / \mathrm{kg} / \mathrm{min}$. TIVA: alfentanil from 1 to $3 \mathrm{mcg} / \mathrm{kg} / \mathrm{min}$ (do not administer boluses) Rocuronium 1 $\mathrm{mg} / \mathrm{kg} / \mathrm{h}$ every $40 \mathrm{~min}$. Sevoflurane 1-2\% in administration. Suspension of anesthesia 10 minutes before the end of the procedure.

\section{Sugammadex}

The administration of sugammadex is provided in all cases in which, after 10 minutes from anesthesia suspension has not had an adequate restoration of spontaneous ventilation; in patients of this weight is not driven TOF because of reliability problems of reading in the premature infant or low weighting patients

a. Failure Intubation: Sugammadex: $16 \mathrm{mg} / \mathrm{kg}$ in 30 seconds with restoration of spontaneous ventilation within 180 seconds. If not resume spontaneous breathing within 180 seconds repeat:
Sugammadex: $6 \mathrm{mg} / \mathrm{kg}$ then: It repeats protocol as monitoring.

b. Extubation Routine: Sugammadex: $3 \mathrm{mg} / \mathrm{kg}$ bolus in a minute, restoration of spontaneous ventilation within 180 seconds.

\section{Patient weight $>10 \mathrm{~kg}<30 \mathrm{~kg}$}

Premedication and research venous access suspend any fasting from 12 hours to suspend the administration of water from 4 hours. Midazolam 0.1 to $0.9 \mathrm{mg} / \mathrm{kg}+$ droperidol 2.5 mg rectally 30 minutes before entering the cath lab if the peripheral venous access is difficult to obtain, induce anesthesia with sevoflurane $3.5-4 \%$ (titrate) do not make more than two attempts to cannulate a peripheral vein or external giugular vein then find a central venous access [12].

\section{Anesthesia}

a. Induction: Midazolam $0.5 \mathrm{mg} / \mathrm{kg}$ in slow bolus Propofol $1 \mathrm{mg} / \mathrm{kg}$ in a minute Rocuronium $1 \mathrm{mg} / \mathrm{kg}$ for induction.

b. Retention: Except for particular cases the maintenance procedures should be started immediately after the arterial cannulation, or immediately in hypertensive patients (eg., aortic coartation) or in patients in whom we proceed to surgical cannulation of the vessels. TIVA: midazolam 2 to $10 \mathrm{mcg} / \mathrm{kg} / \mathrm{min}$ propofol from 1 to $3 \mathrm{mg} / \mathrm{kg} / \mathrm{h}$ (as an alternative to midazolam) Fentanyl 5-15 mcg / kg every 40 minutes. Rocuronium $1 \mathrm{mg} / \mathrm{kg} / \mathrm{h}$ every 40 minutes or TOF guided (value> 2) Sevoflurane 1-2\%. Suspension of anesthesia 10 minutes before the anticipated end of the procedure.

\section{Sugammadex}

The administration of sugammadex is provided in all cases in which, after 10 minutes from anesthesia suspension has not had an adequate RESTORED spontaneous ventilation. TOF tour with $\mathrm{TOF}>2$. TOF not guided with spontaneous breathing in FR> 15 acts minute and End Tidal PCO $2<40 \mathrm{mmHg}$.

a. Failure Intubation: Sugammadex: $16 \mathrm{mg} / \mathrm{kg}$ in 30 seconds with restoration of spontaneous ventilation nea within 180 seconds. If not resume spontaneous breathing within 180 seconds repeat: Sugammadex: $6 \mathrm{mg} / \mathrm{kg}$ then: It repeats protocol as per monitoring.

b. Extubation Routine:Sugammadex: $3 \mathrm{mg} / \mathrm{kg}$ bolus in a minute with restoration of ventilation spontaneous within 180 seconds.

\section{Patient weight $>30 \mathrm{~kg}$}

Premedication and research venous access suspend fasting by 12 hours. Suspend administration of water from 24 hours Temazepam $5 \mathrm{mg}, 1$ tablet per os at 22 [13]. Temazepam 5 $\mathrm{mg}, 1$ tablet by mouth 45 minutes before entering the room hemodynamics. 


\section{Anesthesia}

a. Induction: Midazolam $0.2 \mathrm{mg} / \mathrm{kg}$ bolus. Propofol 1-2 $\mathrm{mg} / \mathrm{kg}$ bolus in a minute Rocuronium $0.9 \mathrm{mg} / \mathrm{kg}$.

b. Retention: The maintenance should be started immediately after induction TIVA: midazolam 2 to $10 \mathrm{mcg} /$ $\mathrm{kg} / \mathrm{min}$. propofol from 1 to $3 \mathrm{mg} / \mathrm{kg} / \mathrm{h}$ (as an alternative to Midazolam) Fentanyl 5-15 mcg / kg every 40 minutes. Rocuronium $0.9 \mathrm{mg} / \mathrm{kg}$ every 40 minutes or TOF guided (value> 2) Sevoflurane 1-2\% in administration. Suspension of anesthesia 10 minutes before the anticipated end of the procedure.

\section{Sugammadex}

The administration of sugammadex is provided in all cases in which, after 10 minutes from anesthesia suspension has not had an adequate RESTORED spontaneous ventilation. TOF tour with TOF $>2$. TOF not guided with spontaneous breathing in FR> 15 acts minute and End Tidal PCO $2<40 \mathrm{mmHg}$.

a. Failure Intubation: Sugammadex: $16 \mathrm{mg} / \mathrm{kg}$ in 30 seconds with restoration of spontaneous ventilation nea within 180 seconds. If not resume spontaneous breathing within 180 secondaryrepeating: Sugammadex: $10 \mathrm{mg} / \mathrm{kg}$ then: It repeats protocol as per monitoring.

\section{Pathology Patients with Type Cyanogen}

\section{Patient weight $<5 \mathrm{~kg}$}

Premedication and research venous access, if you do infuse suspend feeding three hours before the pro- tough you do not need premedication the patient if there is cannulation of the umbilical vein induce anesthesia from such access and continue anesthesia from such access, which will be removed at the end of procedure. If the peripheral venous access is difficult to obtain, induce anesthesia with sevoflurane $3.5-4 \%$ (titrate) do not make more than two attempts to cannulate a peripheral vein or external giugular vein

then find a central venous access [14].

\section{Anesthesia}

a. Induction: If the patient has a history of Tetralogy of Fallot (no s/p MBTS) co-administering immediately after venous cannulation $2 \mathrm{mEq} / \mathrm{kg}$ of NaHCO-3 and 5 to $10 \mathrm{ml}$ / kg of plasma expanders. Midazolam $2 \mathrm{mg}$ in slow bolus. Midazolam additional $1 \mathrm{mg} / \mathrm{kg}$. Ketamine 1-2 mg / kg slowly in at least 4 minutes Rocuronium $1.2 \mathrm{mg} / \mathrm{kg}$.

b. Retention: Except that in the particular maintenance procedures should be started immediately after the arterial cannulation, or immediately in patients in whom proceed surgical cannulation of the vessels. TIVA: midazolam 2 to 8 $\mathrm{mcg} / \mathrm{kg} / \mathrm{min}$. ketamine from 10 to $40 \mathrm{mcg} / \mathrm{kg} / \mathrm{h}$ (nonadministered boluses) Rocuronium $1.2 \mathrm{mg} / \mathrm{kg}$ every 40 minutes. Possible supplementary dose of morphine $0.1 \mathrm{mg} /$ $\mathrm{kg}$ bolus if the pressure blood is unstable or there are serious desaturation Suspension of anesthesia 10 minutes before the anticipated end of the pro- tough.

\section{Sugammadex}

The administration of sugammadex is provided in all cases in which, after 10 minutes from anesthesia suspension has not had an adequate restoration will of spontaneous ventilation and in patients of this weight is not guided TOF date due to reliability problems of reading in pre-baby mature or low weight.

a. Failure Intubation: Sugammadex: $16 \mathrm{mg} / \mathrm{kg}$ in 30 seconds with restoration of spontaneous ventilation nea within 180 seconds. If not resume spontaneous breathing within 180 secondaryrepeating: Sugammadex: $6 \mathrm{mg} / \mathrm{kg}$ then: It repeats protocol as per moniroting.

b. Extubation Routine: Sugammadex $4 \mathrm{mg} / \mathrm{kg}$ bolus in a minute with restoration of ventilation spontaneous within 180 seconds.

\section{Patient Weight $<10 \mathrm{~kg}>5 \mathrm{~kg}$}

Premedication and research venous access suspend any fasting from 12 hours to suspend the administration of water from 4 hours. Midazolam 0.09 to $0.1 \mathrm{mg} / \mathrm{kg}+$ droperidol 1.25 $\mathrm{mg}$ rectally $30 \mathrm{~min}$ - utes you before entering the cath lab if the peripheral venous access is difficult to obtain, induce anesthesia with sevoflurane $3.5-4 \%$ (titrate) do not make more than two attempts to cannulate a peripheral vein or external giugular vein then find a central venous access [15].

\section{Anesthesia}

a. Induction: Midazolam $2 \mathrm{mg}$ Midazolam further slow bolus $1 \mathrm{mg} / \mathrm{kg}$. Ketamine 1-2 mg / kg slowly in at least four minutes Rocuronium $1.2 \mathrm{mg} / \mathrm{kg}$.

b. Retention: Except that in the particular maintenance procedures should be started immediately after the arterial cannulation, or immediately in hypertensive patients (eg., Coarctation of the aorta) or in patients in whom we proceed to surgical cannulation the vessels. Midazolam from 2 to 10 mcg / kg / min Ketamine 1-2 mcg / kg / h. Rocuronium 1 mg / kg every 40 minutes. Sevoflurane $1-2 \%$ in administration supplementary. Suspension of anesthesia 10 minutes before the anticipated end of the procedure.

\section{Sugammadex}

The administration of sugammadex is provided in all cases in which, after 10 minutes from anesthesia suspension has not had an adequate restoration of spontaneous ventilation and in patients of this weight is not TOF guided due to the relative value detected in the patient weighting up to $10 \mathrm{~kg}$.

a. Failure Intubation: Sugammadex: $16 \mathrm{mg} / \mathrm{kg}$ in 30 seconds with restoration of spontaneous ventilation nea within 180 seconds. If not resume spontaneous breathing within 180 seconds repeat: Sugammadex: $6 \mathrm{mg} / \mathrm{kg}$ then: It repeats protocol as per monitoring. 
b. Extubation Routine: Sugammadex: $3 \mathrm{mg} / \mathrm{kg}$ bolus in a minute with restoration of ventilation spontaneous within 180 seconds.

\section{Patient weight $>10 \mathrm{~kg}<30 \mathrm{~kg}$}

Premedication and research venous access suspend any fasting from 12 hours; suspend the administration of water from 4 hours. Midazolam 0.1 to $0.9 \mathrm{mg} / \mathrm{kg}+$ droperidol 2.5 mg rectally 30 minutes before entering the cath lab if the peripheral venous access is difficult to obtain, induce anesthesia with sevoflurane $3.5-4 \%$ (titrate) do not make more than two attempts to cannulate a peripheral vein or external giugular vein

then find a central venous access.

\section{Anesthesia}

a. Induction: Midazolam $0.5 \mathrm{mg} / \mathrm{kg}$ in slow bolus Propofol $1 \mathrm{mg} / \mathrm{kg}$ in a minute Rocuronium $1 \mathrm{mg} / \mathrm{kg}$.

b. Retention: Except that in the particular maintenance procedures should be started immediately after the arterial cannulation, or immediately in hypertensive patients (eg., Coarctation of the aorta) or in patients in whom we proceed to surgical cannulation the vessels. TIVA: ketamine 1-2 mcg / kg / h propofol from 1 to $3 \mathrm{mg} / \mathrm{kg} / \mathrm{h}$ (as an alternative to ketamine) Fentanyl 5-15 mcg / kg every 40 minutes. Rocuronium $1 \mathrm{mg} / \mathrm{kg} / \mathrm{h}$ every 40 minutes or TOF guided (value $>2$ ) Sevoflurane 1-2\%. Suspension of anesthesia 10 minutes before the anticipated end of the procedure.

\section{Sugammadex}

The administration of sugammadex is provided in all cases in which, after 10 minutes from anesthesia suspension has not had an adequate RESTORED spontaneous ventilation. TOF tour with TOF $>2$. TOF not guided with spontaneous breathing in FR> 15 acts minute and End Tidal PCO $2<40 \mathrm{mmHg}$.

a. Failure Intubation: Sugammadex: $16 \mathrm{mg} / \mathrm{kg}$ in 30 seconds with restoration of spontaneous ventilation within 180 seconds. If not resume spontaneous breathing within 180 seconds repeati:

Sugammadex: $6 \mathrm{mg} / \mathrm{kg}$ then: It repeats protocol as per monitoring.

b. Extubation Routine: Sugammadex: $3 \mathrm{mg} / \mathrm{kg}$ bolus in a minute with restoration of ventilation spontaneous within 180 seconds.

\section{Patient weight $>30 \mathrm{~kg}$}

Premedication and research venous access, suspend fasting by 12 hours suspend administration of water from 4 hours Temazepam $5 \mathrm{mg}$, 1 tablet per os at 22. Temazepam $5 \mathrm{mg}, 1$ tablet by mouth 45 minutes before entering the Meeting operating.

\section{Anesthesia}

a. Induction: Midazolam $0.2 \mathrm{mg} / \mathrm{kg}$ bolus. Propofol 1-2 $\mathrm{mg} / \mathrm{kg}$ bolus in a minute Rocuronium $0.9 \mathrm{mg} / \mathrm{kg}$.

b. Retention: The maintenance should be started immediately after induction Propofol TIVA from 1 to 3 mg / kg / h. Fentanyl 5-15 mcg / kg every 40 minutes. Rocuronium $0.9 \mathrm{mg} / \mathrm{kg}$ every 40 minutes or TOF guided (value $>2$ ) Sevoflurane 1-2\% in administration. Suspension of anesthesia 10 minutes before the anticipated end of the procedure.

\section{Sugammadex}

The administration of sugammadex is provided in all cases in which, after 10 minutes from anesthesia suspension has not had an adequate RESTORED spontaneous ventilation. TOF tour with TOF $>2$. TOF not guided with spontaneous breathing in FR> 15 acts minute and End Tidal PCO $2<40 \mathrm{mmHg}$.

a. Failure Intubation: Sugammadex: $16 \mathrm{mg} / \mathrm{kg}$ in 30 seconds with restoration of spontaneous ventilation nea within 180 seconds. If not resume spontaneous breathing within 180 secondaryrepeating: Sugammadex: $10 \mathrm{mg} / \mathrm{kg}$ then: It repeats protocol as per monitoring.

\section{References}

1. Milella L, Da Cruz E, Gajraj RJ, Corno AF (1997) Computerized Anaesthesia and Normothermic perfusion in paediatric cardiac surgery. The Second World Congress of Pediatric Cardiology and Cardiac Surgery Honolulu, Hawaii, May, p. 11-15.

2. Milella L, Mele D (1998) TCI: preliminary experience in paediatric cardiac anaesthesia. Italy.

3. Milella L (1999) TCI in Paediatric Cardiac Anaesthesia. In: Milella L (Eds.), CARACT 99, $\mathrm{V}^{\text {th }}$ Course in Cardiothoracic Anaesthesia, Milan. Italy.

4. Milella L (2000) Advances in Pediatric Cardiovascular Anesthesia. IX ${ }^{\text {th }}$ Congress of Latin American Society of Cardiology and Cardiac Surgery. USA.

5. Milella L, Calabrese G (2000) TCI in Pediatric Cardiac Anesthesia: a better choice for early extubation? IX ${ }^{\text {th }}$ Congress of Latin American Society of Cardiology and Cardiac Surgery. USA.

6. Milella L, Calabrese G, Fiore T (2005) TCI in Paediatric Cardiac Anesthesia: 59 cases. Minerva Anestesiologica.

7. Milella L, Cassano V (2007) Warm Surgery: our experience. Eur J Cardiothorac surg 31(4): 754-755.

8. Milella L (2014) Anaesthesia protocols in paediatric cardiac surgery: the use of sugammadex-Patients and surgical Procedures. p. 31-43.

9. Milella L (2014) Anaesthesia protocols in paediatric diagnostic and interventional cath lab procedures: the use of sugammadex-Patients and Emodinamic Procedures. p. 44-55.

10. Milella L (2015) Anaesthesia protocols in paediatric video thoraco laparoscopic surgical procedures: the use of rocuronium bromide and sugammadex. p. 41-54. 
11. Milella L (2015) $69^{\circ}$ th Italian National Anaesthesia Society Congress (SIAARTI): Paediatric Anaesthesia: Paediatric TIVA-TCI: Tips and Pricks. Italy.

12. Milella L (2017) Teaching Project for analgesic and sedation therapeutics in pediatric patient in ICU. Italy.

13. Milella L (2018) Journal of Pediatrics and Neonatal Care: Neonatal and Pediatric General and Cardiac Anesthesia and ICU: wath"s new in 2017/2018? -Bari Pediatric Hospital Experience-Italy. 8(2).
14. Milella L (2018) Anesthesia Control: A Personal Opinion. Curr Trends Biomedical Engineering \& Biosciences 12(1)

15. Bech L, Milella L, Labellarte C (2018) Continuous non-invasive measurement of stroke volume and cardiac output in infants and children: comparison of Impedance Cardiography NICaS $®$ vs Cardio Q® method. Clin Ter 169(3): e110-113.

Your next submission with Juniper Publishers will reach you the below assets

- Quality Editorial service

- Swift Peer Review

- Reprints availability

- E-prints Service

- Manuscript Podcast for convenient understanding

- Global attainment for your research

- Manuscript accessibility in different formats

(Pdf, E-pub, Full Text, Audio)

- Unceasing customer service

Track the below URL for one-step submission https://juniperpublishers.com/online-submission.php 